\title{
Analysis of Adaptive Interference Cancellation Using Common-Mode Information in Wireline Communications
}

\author{
Thomas Magesacher, Per Ödling, and Per Ola Börjesson \\ Department of Information Technology, Lund University, P.O. Box 118, 22100 Lund, Sweden
}

Received 4 September 2006; Accepted 1 June 2007

Recommended by Ricardo Merched

Joint processing of common-mode (CM) and differential-mode (DM) signals in wireline transmission can yield significant improvements in terms of throughput compared to using only the DM signal. Recent work proposed the employment of an adaptive CM-reference-based interference canceller and reported performance improvements based on simulation results. This paper presents a thorough investigation of the cancellation approach. A subchannel model of the CM-aided wireline channel is presented and the Wiener solutions for different adaptation strategies are derived. It is shown that a canceller, whose coefficients are adapted while the far-end transmitter is silent, yields a signal-to-noise power ratio (SNR) that is higher than the SNR at the DM channel output for a large class of practically relevant cases. Adaptation while the useful far-end signal is present yields a front-end whose output SNR is considerably lower compared to the SNR of the DM channel output. The results are illustrated by simulations based on channel measurement data.

Copyright (c) 2007 Thomas Magesacher et al. This is an open access article distributed under the Creative Commons Attribution License, which permits unrestricted use, distribution, and reproduction in any medium, provided the original work is properly cited.

\section{INTRODUCTION}

Transmission of information over copper cables is conventionally carried out by differential signalling. On physicallayer level, this corresponds to the application of a voltage between the two wires of a pair. The signal at the receive side is derived from the voltage measured between the two wires. Differential-mode (DM) signalling over twisted-wire pairs, originally patented by Bell more than hundred years ago [1], exhibits a high degree of immunity against ingress of unwanted interference, caused, for example, by radio transmitters (radio frequency interference) or by data transmission in neighboring pairs (crosstalk) [2]. The inherent immunity of a cable against ingress decays with frequency. In fact, the performance of almost all high data-rate (and thus also highbandwidth consuming) digital subscriber line (DSL) systems is limited by crosstalk.

The number of strong crosstalk sources is often very low-one, two or three dominant crosstalkers significantly raise the crosstalk level and thus reduce the performance on the pair under consideration. In such cases, it is beneficial to exploit the common-mode (CM) signal, which is the signal corresponding to the arithmetic mean of the two voltages measured between each wire and earth, at the receive side
[3-5]. The CM signal and the DM signal of a twisted-wire pair are strongly correlated. Exploiting the CM signal in addition to the DM signal yields a new channel whose capacity can be, depending on the scenario, up to about three times higher than the conventional DM-only channel capacity [3]. The large benefit is achieved for exactly those scenarios that are challenged by strong interference. The additional receive signal yields an additional degree of freedom, which can be exploited to mitigate interference.

This paper investigates the receiver front-end for CMaided wireline transmission. Independent work proposed the use of an interference canceller consisting of a linear adaptive filter fed by the CM signal $[6,7]$. Adaptive processing of correlated receive signals bears the potential danger of cancelling the useful component. Despite the performance improvements reported in $[6,7]$, it is a priori not clear whether this kind of adaptive interference cancellation is beneficial or counterproductive.

In the following, a more rigorous approach is pursued. Section 2 introduces a suitable channel model in frequency domain, which allows us to carry out the analysis on subchannel level. Based on experience gained from measurements, some channel characteristics which hold for a large class of practical scenarios are identified in Section 3. 
In Section 4, the maximum likelihood (ML) estimator of the transmit signal is derived. The ML estimator suggests a receiver front-end which has the structure of a linear interference canceller with coefficients adjusted so that the signalto-noise power ratio (SNR) at the canceller output is maximised. The performance of adaptive cancellation is analysed by means of Wiener filter solutions. Section 5 illustrates the results through performance simulations based on channel measurements. Section 6 concludes the work.

\section{SYSTEM MODEL}

The wireline channel can be modelled as a linear stationary Gaussian channel with memory and coloured interference (correlated in time). In general, interference originates from an arbitrary number $S$ of sources, which typically model far-end crosstalk (FEXT) and near-end crosstalk (NEXT) in a multipair cable [2]. We choose to model the channel in frequency domain for two reasons. First, frequency-domain modelling yields valuable insights and supports a simple analysis based on subchannels. Second, a frequency-domain model is the natural choice considering that most modern wireline systems are based on multicarrier modulation. The application of the suggested subchannel interference canceller in multicarrier systems is thus straightforward.

The DM output $Y_{1}[m]$ and the CM output $Y_{2}[m]$ of a twisted-wire pair at the $m$ th subchannel can be written as

$$
\begin{aligned}
& {\left[\begin{array}{c}
Y_{1}[m] \\
Y_{2}[m]
\end{array}\right]=\left[\begin{array}{l}
a[m] \\
b[m]
\end{array}\right] X[m]} \\
& +\left[\begin{array}{llllcc}
c_{1}[m] & c_{2}[m] & \cdots & c_{S}[m] & n_{1}[m] & 0 \\
d_{1}[m] & d_{2}[m] & \cdots & d_{S}[m] & 0 & n_{2}[m]
\end{array}\right]\left[\begin{array}{c}
Z_{1}[m] \\
Z_{2}[m] \\
\vdots \\
Z_{S}[m] \\
N_{1}[m] \\
N_{2}[m]
\end{array}\right]
\end{aligned}
$$

for $0 \leq m \leq M-1$, where $M$ is the number of subchannels. The choice of $M$ may be influenced by the parameters of the wireline system the interference canceller is applied to. An obvious choice for $M$ is the system's number of tones. Hereinafter, we omit the subchannel index $m$ wherever possible for the sake of simple notation. $X, N_{1}, N_{2}$, and $Z_{i}, 1 \leq i \leq S$, are mutually independent, zero-mean, unit-variance, complex, circularly symmetric Gaussian random variables. $X$ is the far-end transmit signal. $N_{1}$ and $N_{2}$ model background noise present at the wire-pair's output ports of DM and CM, respectively. The $S$ interference sources are modelled by $Z_{i}$, $1 \leq i \leq S$.

The complex coefficients $a \in \mathbb{C}$ and $b \in \mathbb{C}$ model the coupling from the far-end DM port to the DM port and to the CM port, respectively. The coefficients $c_{i} \in \mathbb{C}$ and $d_{i} \in \mathbb{C}$ model the coupling from the $i$ th interference source to the DM port and to the CM port, respectively. The coefficients $n_{1} \in \mathbb{C}$ and $n_{2} \in \mathbb{C}$ scale and colour the background noise present at the DM port and at the CM port, respectively. Figure 1 depicts a block diagram of this frequency-domain

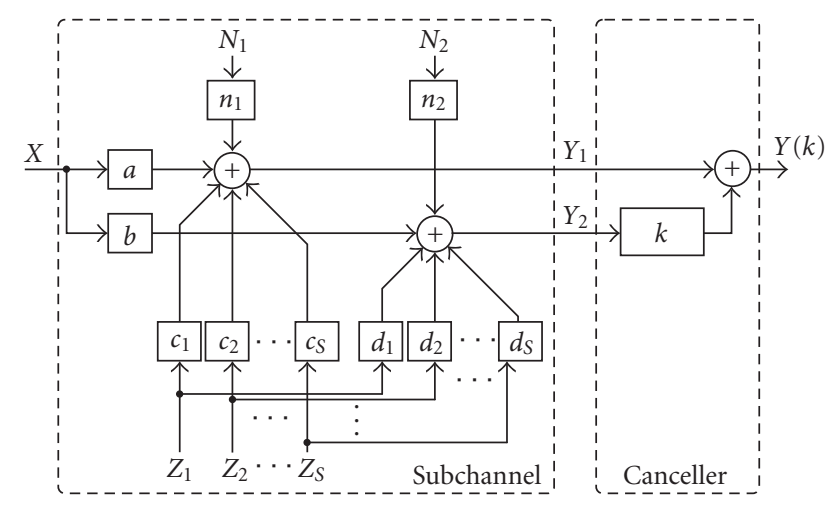

FIGURE 1: Model of the subchannel (1) and the corresponding scalar linear interference canceller (8).

model, which allows us to continue the analysis on subchannel level.

\section{CHANNEL PROPERTIES}

Based on cable models $[2,8]$ and on experience from measurements $[4,9]$, we observe that a large number of practically relevant scenarios obey the following conditions $(|\cdot|$ denotes absolute value):

Assumption 1. $|a| \stackrel{(\alpha)}{\gg}\left|c_{i}\right| \stackrel{(\beta)}{\approx}|b| \stackrel{(\gamma)}{\approx}\left|d_{j}\right| \stackrel{(\delta)}{\gg}\left|n_{2}\right| \stackrel{(\epsilon)}{\approx}\left|n_{1}\right|, i, j \quad \in$ $\{1, \ldots, S\}$.

For FEXT, $(\alpha)$ always holds since the model for the FEXT coupling function includes scaling by the insertion loss of the line. For NEXT, in systems with overlapping frequency bands for upstream and downstream, $(\alpha)$ does not necessarily hold for long loops and/or high frequencies since, at least according to the ETSI model [8], the NEXT coupling function is not scaled by the insertion loss and is thus independent of the loop length. Consequently, the level of the receive signal power spectral density (PSD) on long loops may be lower than the NEXT PSD level. Most high-bandwidth consuming DSLs, however, employ frequency division duplexing and are thus only vulnerable to alien NEXT, that is, NEXT from systems of different types, and "out-of-band self-NEXT," that is, NEXT caused by the out-of-band transmit signals of systems of the same type. Alien NEXT is often taken care of by spectral management. Self-NEXT is usually negligible due to out-of-band spectral masks. The CM-related assumptions $(\beta)$ and $(\gamma)$ are mainly based on measurement experience $[4,9]$. While $(\delta)$ always holds for NEXT, it may not be true for FEXT on long loops, where the FEXT PSD level may lie below the PSD level of the background noise due to the loop attenuation. Assumption $(\epsilon)$ states that the CM background noise level is of the same order of magnitude as the DM background noise level.

To conclude, Assumption 1 is valid for frequency division duplexed systems as long as the pair under consideration and the crosstalk-causing pair have roughly the same length and are neither extremely short nor extremely long. In case the 


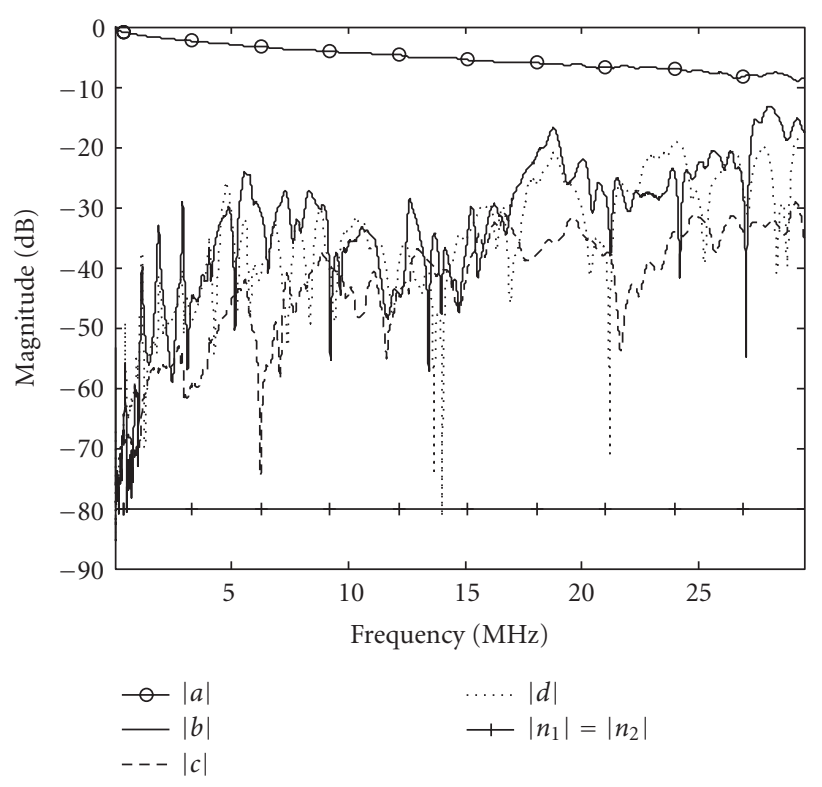

FIgURE 2: Channel properties $a, b, c, d$ obtained from measurements. The $y$-axis denotes relative magnitude in $\mathrm{dB}$ (the raw results are normalised by the magnitude of the largest $a$-value). Assuming a VDSL transmit PSD of $-60 \mathrm{dBm} / \mathrm{Hz}$ results in a level of $-80 \mathrm{~dB}$ for $n_{1}$ and $n_{2}$ in order to obtain a background-noise PSD of $-140 \mathrm{dBm} / \mathrm{Hz}$, which is the level suggested in standardisation documents $[8,10]$.

pairs are extremely short, the crosstalk PSD levels are very low and consequently $(\beta)$ does not hold. In case the pairs are extremely long, both the crosstalk PSD levels and the receive signal PSD levels are very low, which may lead to neither $(\alpha)$ nor $(\beta)$ being true. Cases with extreme lengths (short or long) are of little practical interest, since extremely short loops are not found in the field and extremely long loops are out of scope for high-bandwidth consuming DSL techniques. Care should be taken with near/far scenarios for which $(\alpha)$ does not necessarily hold since the useful signal is severely attenuated while the crosstalk is strong.

Figure 2 shows exemplary channel transfer and coupling functions based on measurements [4]. The magnitude values are normalised by the magnitude of the largest measurement result for the transfer function. Assuming a VDSL transmit PSD of $-60 \mathrm{dBm} / \mathrm{Hz}$ and a background-noise PSD of $-140 \mathrm{dBm} / \mathrm{Hz}$, which is the level suggested in standardisation documents $[8,10]$, results in a level of $-80 \mathrm{~dB}$ for $n_{1}$ and $n_{2}$. Assumption 1 holds over nearly the whole frequency range for the channel measurements depicted in Figure 2.

\section{ANALYSIS}

\subsection{Maximum likelihood (ML) estimator}

The linear Gaussian model (1) of a subchannel can be written as

$$
\underbrace{\left[\begin{array}{l}
Y_{1} \\
Y_{2}
\end{array}\right]}_{\hat{=} \boldsymbol{Y}} \hat{=} \underbrace{\left[\begin{array}{l}
a \\
b
\end{array}\right]}_{\hat{=} \boldsymbol{H}} X+\boldsymbol{V},
$$

where the vector $V$ contains both noise and interference. The covariance matrix $\boldsymbol{C}_{\mathrm{v}}$ of $\boldsymbol{V}$ is given by

$$
\boldsymbol{C}_{\mathrm{v}} \hat{=} \mathrm{E}\left(\boldsymbol{V} \boldsymbol{V}^{\mathrm{H}}\right)=\boldsymbol{H}_{\mathrm{v}} \boldsymbol{H}_{\mathrm{v}}^{\mathrm{H}}, \quad \boldsymbol{H}_{\mathrm{v}} \hat{=}\left[\begin{array}{cccccc}
c_{1} & c_{2} & \cdots & c_{S} & n_{1} & 0 \\
d_{1} & d_{2} & \cdots & d_{S} & 0 & n_{2}
\end{array}\right],
$$

where $\mathrm{E}(\cdot)$ and $\cdot \mathrm{H}$ denote expectation and Hermitian transpose, respectively. Note that $a, b, c, d, n_{1}$, and $n_{2}$ are complexvalued. The ML estimator of $X$ is defined as [11]

$$
\widehat{X} \hat{=} \arg \max _{X} f(\boldsymbol{Y} \mid X)
$$

where $f(\boldsymbol{Y} \mid X)$ denotes the likelihood of $X$ (probability density function of $\boldsymbol{Y}$ given $X)$. For the linear Gaussian model (2), the ML estimator can be written as [11]

$$
\widehat{X}=\left(\boldsymbol{H}^{\mathrm{H}} \boldsymbol{C}_{\mathrm{v}}^{-1} \boldsymbol{H}\right)^{-1} \boldsymbol{H}^{\mathrm{H}} \boldsymbol{C}_{\mathrm{v}}^{-1} \boldsymbol{Y} .
$$

Inserting (2) and (3) into (5) followed by mostly straightforward calculus yields

$$
\hat{X}=\rho\left(k_{\mathrm{ML} 1} Y_{1}+k_{\mathrm{ML} 2} Y_{2}\right)=\rho k_{\mathrm{ML} 1} \underbrace{(Y_{1}+\underbrace{\frac{k_{\mathrm{ML} 2}}{k_{\mathrm{ML} 1}} Y_{2}}_{\hat{=} k_{\mathrm{ML}}})}_{\hat{=} Y\left(k_{\mathrm{ML}}\right)}
$$

with

$$
\begin{aligned}
& \rho= \\
& \frac{1}{\left(\sum_{i}\left|d_{i}\right|^{2}+\left|n_{2}\right|^{2}\right)|a|^{2}+\left(\sum_{i}\left|c_{i}\right|^{2}+\left|n_{1}\right|^{2}\right)|b|^{2}-2 \operatorname{Re}\left(a b^{*} \sum_{i} c_{i}^{*} d_{i}\right)}, \\
& k_{\mathrm{ML} 1}=a^{*}\left(\sum_{i}\left|d_{i}\right|^{2}+\left|n_{2}\right|^{2}\right)-b^{*} \sum_{i} c_{i}^{*} d_{i}, \\
& k_{\mathrm{ML} 2}=b^{*}\left(\sum_{i}\left|c_{i}\right|^{2}+\left|n_{1}\right|^{2}\right)-a^{*} \sum_{i} c_{i} d_{i}^{*},
\end{aligned}
$$

where $\operatorname{Re}(\cdot)$ and $*$ denote real part and complex conjugate, respectively.

The ML solution (6) suggests a linear combination of $Y_{1}$ and $Y_{2}$ as estimator, which essentially corresponds to linear interference cancellation depicted in Figure 1 and described by

$$
Y(k)=Y_{1}+k Y_{2}
$$

Choosing $k=k_{\mathrm{ML}}=k_{\mathrm{ML} 2} / k_{\mathrm{ML} 1}$ and applying the scaling factor $\rho k_{\mathrm{ML} 1}$ to the output of the canceller realises the ML solution. The mutual information between $X$ and canceller output $Y(k)$, when the subchannel canceller is adjusted to the coefficient $k$, can be written as [12]

$$
\mathrm{I}(X ; Y(k))=\log (1+\operatorname{SNR}(k)),
$$


where the subchannel SNR at the canceller output is given by

$$
\operatorname{SNR}(k)=\frac{|a+b k|^{2}}{\sum_{i}\left|c_{i}+d_{i} k\right|^{2}+\left|n_{1}\right|^{2}+\left|n_{2} k\right|^{2}} .
$$

Note that $k_{\mathrm{ML}}$ is the interference canceller coefficient for which the mutual information $\mathrm{I}\left(X ; Y\left(k_{\mathrm{ML}}\right)\right)$ is maximised. Furthermore, $\mathrm{I}\left(X ; Y\left(k_{\mathrm{ML}}\right)\right)$ is equal to the mutual information $\mathrm{I}\left(X ; Y_{1}, Y_{2}\right)$ of the transmit signal $X$ and the receive signal pair $\left(Y_{1}, Y_{2}\right)$. In other words, the ML-based canceller preserves all the information contained in the two channel output signals.

\subsection{Steady-state performance of adaptive cancellation}

CM-aided reception can be applied in autonomous receivers and does not require cooperation with receivers of adjacent lines. Thus, CM-aided reception can be used to complement or enhance level-2 or level-3 dynamic spectrum management proposals [13], which rely on colocated receivers. Unlike in many other applications, the ML receiver is not too complex for implementation; however, it requires perfect knowledge of the channel and of the statistics of noise and interference. Since this knowledge is often not available, receiver structures that operate without any kind of side information are of great practical importance. In the following, the suitability of adaptive cancellation schemes based on a squared error criterion is investigated. Popular examples of such schemes are the least-mean square (LMS) and the recursive least squares (RLS) algorithm. In a stationary environment, these algorithms can be parametrised in such a way that they converge towards the Wiener filter solution [14].

In general, the Wiener filter minimises the cost function defined as the mean of the squared error. In our setup, this corresponds to minimising the energy of the interference canceller's output signal $Y(k)$ given by $(8)$ with respect to $k$. The Wiener filter solution $k_{\mathrm{W}}$ is defined by [14]

$$
k_{\mathrm{W}} \hat{=} \arg \min _{k} \mathrm{E}\left(|Y(k)|^{2}\right) .
$$

For our interference canceller model (8), the Wiener filter can be expressed as (cf. Appendix A)

$$
k_{\mathrm{W}}=-\frac{\mathrm{E}\left(Y_{1} Y_{2}^{*}\right)}{\mathrm{E}\left(Y_{2} Y_{2}^{*}\right)}
$$

In the following, we distinguish between the Wiener filter solution $k_{\mathrm{W} 1}$ obtained for $X \neq 0$ and the Wiener filter solution $k_{\mathrm{W} 2}$ obtained for $X=0$. Inserting (2) and (3) into (12), we obtain

$$
k_{\mathrm{W} 1}=-\frac{\mathrm{E}\left(Y_{1} Y_{2}^{*}\right)}{\mathrm{E}\left(Y_{2} Y_{2}^{*}\right)}=-\frac{a b^{*}+\sum_{i} c_{i} d_{i}^{*}}{|b|^{2}+\sum_{i}\left|d_{i}\right|^{2}+\left|n_{2}\right|^{2}},
$$

which is the solution a properly parameterised algorithm converges to when the coefficients are adapted while the useful transmit signal is present. For $X=0$, we obtain

$$
\begin{aligned}
k_{\mathrm{W} 2} & =\arg \min _{k} \mathrm{E}\left(\left.|Y(k)|^{2}\right|_{X=0}\right)=-\left.\frac{\mathrm{E}\left(Y_{1} Y_{2}^{*}\right)}{\mathrm{E}\left(Y_{2} Y_{2}^{*}\right)}\right|_{X=0} \\
& =-\frac{\sum_{i} c_{i} d_{i}^{*}}{\sum_{i}\left|d_{i}\right|^{2}+\left|n_{2}\right|^{2}},
\end{aligned}
$$

which is the solution a properly parameterised algorithm converges to when the coefficients are adapted while there is no useful transmit signal.

As a reference when assessing the performance of adaptive algorithms, we will use the mutual information between $X$ and $Y_{1}$, which can be written as

$$
\mathrm{I}\left(X ; Y_{1}\right)=\log \left(1+\mathrm{SNR}_{\mathrm{DM}}\right)
$$

where the DM-subchannel SNR is given by

$$
\mathrm{SNR}_{\mathrm{DM}}=\frac{|a|^{2}}{\sum_{i}\left|c_{i}\right|^{2}+\left|n_{1}\right|^{2}} \text {. }
$$

\subsection{Implications of Assumption 1 on the steady-state performance of adaptive cancellation}

Under Assumption 1, it can be shown that the following two propositions hold. Instead of proofs, which are merely technical (cf. Appendix B), we provide here motivations for the propositions, which are more insightful and simple to follow.

Proposition 1. Under the conditions defined in Assumption 1, the following inequality holds:

$I\left(X[m] ; Y\left(k_{W 1}[m]\right)\right) \leq I\left(X[m] ; Y_{1}[m]\right), \quad 0 \leq m \leq M-1$.

In other words, in each subchannel, the SNR of the output $Y\left(k_{W 1}\right)$ of a linear interference canceller with tap setting $k_{W 1}$ given by (13) is lower than the SNR of $Y_{1}$.

\section{Motivation}

Since the strongest component in $Y_{1}$ stems from $X$, there is a mechanism driving the canceller coefficient towards $-a / b$, which is the coefficient that eliminates $X$ (note that $|a / b| \gg$ 1). Since increasing $|k|$ increases the residual of $Z$ in $Y(k)$, there is a counter mechanism working against large values of $|k|$. These two mechanisms reach an equilibrium for the solution given by (13). As a net result, the power of $X$ in $Y\left(k_{\mathrm{W} 1}\right)$ is reduced (compared to $Y_{1}$ ), which implies $\left|k_{\mathrm{W} 1}\right| \gg 1$. However, the larger $\left|k_{\mathrm{W}_{1}}\right|$, the higher the power of the $Z$ component in $Y\left(k_{\mathrm{W} 1}\right)$. More precisely, for any $k_{\mathrm{W} 1}$ that fulfils $\left|k_{\mathrm{W} 1}\right|>2$, the power of the $Z$-component in $Y\left(k_{\mathrm{W}_{1}}\right)$ is higher than in $Y_{1}$. To summarise, while the power of the $X$ component is lower in $Y\left(k_{\mathrm{W} 1}\right)$ than in $Y_{1}$, the power of the $Z$-component is higher in $Y\left(k_{\mathrm{W} 1}\right)$ than in $Y_{1}$, which confirms Proposition 1. The proof is given in Appendix B. 
Remark 1. In case there is no dominant interference $Z$, which corresponds in our setting to $c=d=0$, adaptation while $X \neq 0$ yields $k_{\mathrm{W} 1} \approx-a / b$, which essentially eliminates $X$.

Proposition 2. Under the conditions defined in Assumption 1, the following inequality holds:

$I\left(X[m] ; Y\left(k_{W 2}[m]\right)\right) \geq I\left(X[m] ; Y_{1}[m]\right), \quad 0 \leq m \leq M-1$.

In other words, in each subchannel, the SNR of the output $Y\left(k_{W 2}\right)$ of a linear interference canceller with tap setting $k_{W 2}$ given by (14) is higher than the SNR of $Y_{1}$.

\section{Motivation}

When the far-end transmitter is silent $(X=0)$, the strongest component in $Y_{1}$ stems from $Z$. Then, the Wiener filter solution is close to $-c / d$ (the exact solution is given by (14)), which essentially eliminates $Z$. Since $\left|k_{\mathrm{W} 2}\right| \approx|c / d| \approx 1$, the power of the $N_{2}$-component in $Y\left(k_{\mathrm{W} 2}\right)$ remains negligible. A lower and an upper bound on the signal energy (i.e., energy of $X)$ contained in $Y\left(k_{\mathrm{W} 2}\right)$ are $|a|^{2}-|b|^{2}$ and $|a|^{2}+|b|^{2}$, respectively. Consequently, the front-end causes a negligible reduction of signal power $(|b| \ll|a|)$ while essentially eliminating the interference. Thus, its performance is close to that of the ML estimator. The proof of Proposition 2 is given in Appendix B.

Remark 2. In case there is no dominant interference $Z$ ( $c=$ $d=0$ ), adaptation with $X=0$ yields $k_{\mathrm{W} 2}=0$, which is close to the ML solution $b^{*}\left|n_{1}\right|^{2} / a^{*}\left|n_{2}\right|^{2}$.

The conclusion drawn from Propositions 1 and 2 for a typical wireline scenario (typical in the sense that Assumption 1 is valid) with one dominant crosstalker is the following: a canceller set to the Wiener filter solution $k_{\mathrm{W} 2}$ (i.e., when adaptation is performed while the transmitter is silent) exhibits a higher SNR at the output compared to the DM channel output. Moreover, the performance is close to the ML estimator's performance. A canceller set to the Wiener filter solution $k_{\mathrm{W} 1}$ (i.e., when adaptation is performed while the transmitter is active) exhibits a lower SNR at the canceller output compared to the DM channel output.

Note that Propositions 1 and 2 hold for the interferencecanceller front-end (8) set to the corresponding Wiener-filter solution. The results might not be valid for more advanced receivers that, for example, jointly decode and estimate the channel.

\subsection{Impact of coefficient mismatch on steady-state performance}

The design of adaptive algorithms that converge to the Wiener filter solution involves a tradeoff between convergence time and mismatch. In general, the faster an adaptive algorithm reaches a steady solution, the larger the deviation from the desired Wiener filter solution becomes [14]. Hereinafter, we focus on the mismatch of a canceller adapted while $X=0$, that is, its mismatch with respect to $k_{\mathrm{W} 2}$. In order to assess the sensitivity of the achieved SNR with respect to the mismatch, we quantify this mismatch in terms of the relative deviation of the coefficient's absolute value. A mismatch of up to $10 \%$, for example, is expressed as $\left|\left(k-k_{\mathrm{W} 2}\right) / k_{\mathrm{W} 2}\right| \leq 0.1$. We denote the set of coefficients with a mismatch of up to $\mu$ as

$$
\mathcal{K}_{\mu} \hat{=}\left\{k:\left|\left(k-k_{\mathrm{W} 2}\right) / k_{\mathrm{W} 2}\right| \leq \mu\right\}
$$

and the corresponding set of $\operatorname{SNR}$ values as $\operatorname{SNR}\left(\mathcal{K}_{\mu}\right)$. The SNR is not necessarily a rotationally symmetric function of real part and imaginary part of $k$ around the peak corresponding to $k_{\mathrm{ML}}$. The sensitivity of the SNR with respect to $k$ depends on the channel coefficients. Figure 3 depicts two examples: while the SNR decay is in the same order of magnitude for all directions in Figure 3(a), the sensitivity of the SNR along the direction corresponding to the imaginary part is negligible in Figure 3(b). The coefficients in the set $\mathcal{K}_{\mu}$ lie inside or on the marked circle $\left\{k:\left|\left(k-k_{\mathrm{W} 2}\right) / k_{\mathrm{W} 2}\right|=\mu\right\}$. The worst-case SNR is obtained for one or more coefficients on the circle. In the examples presented in the following section, the sensitivity of the performance with respect to the coefficient's mismatch is quantified in terms of $\operatorname{SNR}\left(\mathcal{K}_{\mu}\right)$.

\section{SIMULATION RESULTS}

In order to illustrate the implications of the propositions presented in the previous section, we evaluate the performance of adaptive cancellation in terms of the SNR at the canceller output given by (10). For comparison, the SNR of DM-only processing, given by (16), and the SNR of the ML estimator are computed. We consider $M=8192$ subchannels in the frequency range from $3 \mathrm{kHz}$ to $30 \mathrm{MHz}$. The coupling functions are obtained from cable measurements [4] using the lengthadaptation methods suggested in [3].

\subsection{Example 1: equal-length FEXT}

We begin with a transmission scenario over a loop of length $300 \mathrm{~m}$. We assume a flat transmit PSD of $-60 \mathrm{dBm} / \mathrm{Hz}$ and flat noise PSDs of $-140 \mathrm{dBm} / \mathrm{Hz}$ at both the $\mathrm{CM}$ port and the DM port of the receiver. Furthermore, there is one crosstalk source $(S=1)$ located at the same distance and transmitting with the same PSD as the transmitter. The results for this scenario, depicted in Figure 4, agree with the propositions presented in the previous section. Adaptation in the absence of the far-end signal yields a signal-to-noise ratio $\operatorname{SNR}\left(k_{\mathrm{W} 2}\right)$ that exceeds the signal-to-noise ratio $\mathrm{SNR}_{\mathrm{DM}}$ achieved by DM-only processing for virtually the whole frequency range. Moreover, $\operatorname{SNR}\left(k_{\mathrm{W} 2}\right)$ is virtually the same as the upper limit given by $\operatorname{SNR}\left(k_{\mathrm{ML}}\right)$. Adaptive interference cancellation eliminates the crosstalk almost completely. The resulting SNR is merely limited by the background noise. Consequently, the performance is sensitive to a mismatch of the canceller coefficients. A mismatch of $10 \%$ can result in a performance degradation of up to $8 \mathrm{~dB}$ for sensitive subchannels. Adaptation in the presence of the far-end signal, on the other hand, yields a signal-to-noise ratio $\operatorname{SNR}\left(k_{\mathrm{W} 1}\right)$ that is much lower than $\mathrm{SNR}_{\mathrm{DM}}$ over the whole frequency range. 


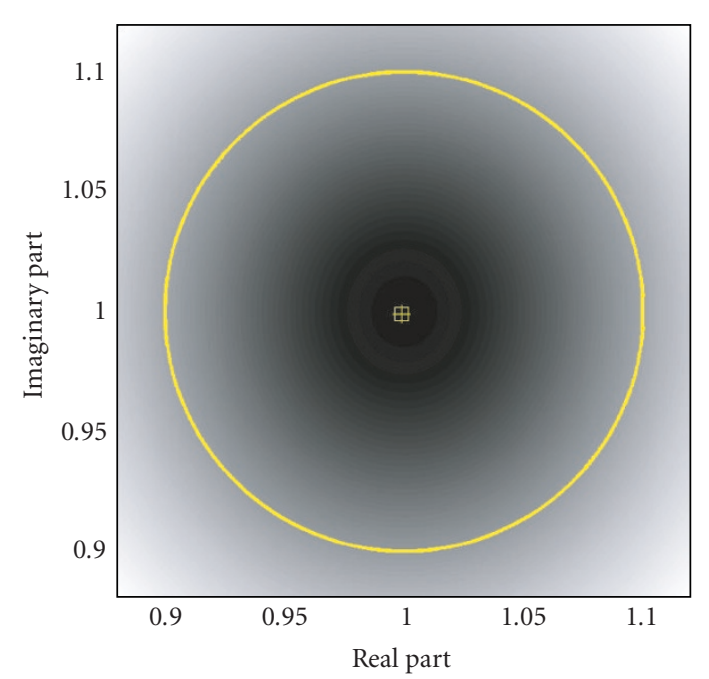

(a)

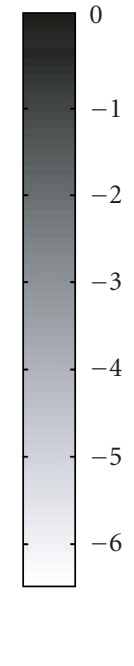

FIGURE 3: Normalised SNR $10 \log _{10}\left(\operatorname{SNR}(k) / \mathrm{SNR}\left(k_{\mathrm{W} 2}\right)\right)$ in $\mathrm{dB}$ as a function of real part and imaginary part of $k / k_{\mathrm{W} 2}$ for two different choices of channel coefficients $a, b, c, d, n_{1}, n_{2}$. While the SNR decay is in the same order of magnitude for all directions for case (a), the sensitivity of the SNR along the direction corresponding to the imaginary part is negligible for case (b). Coefficients with a mismatch of up to $10 \%$, denoted by the set $\mathcal{K}_{0.1}$, lie inside or on the marked circle. The plus-marker indicates $k_{\mathrm{W} 2}$ and the square-marker indicates $k_{\mathrm{ML}}$.
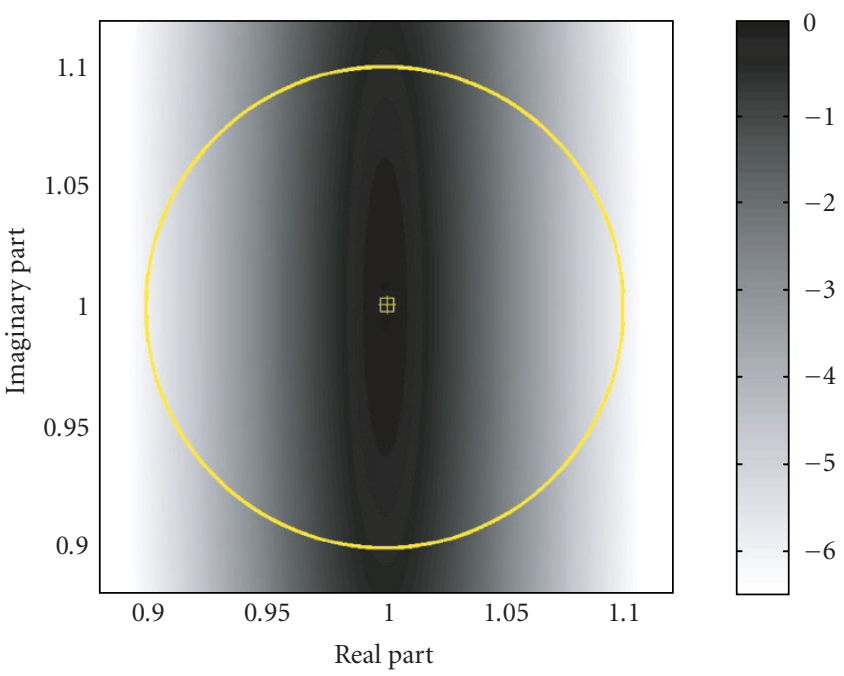

(b)

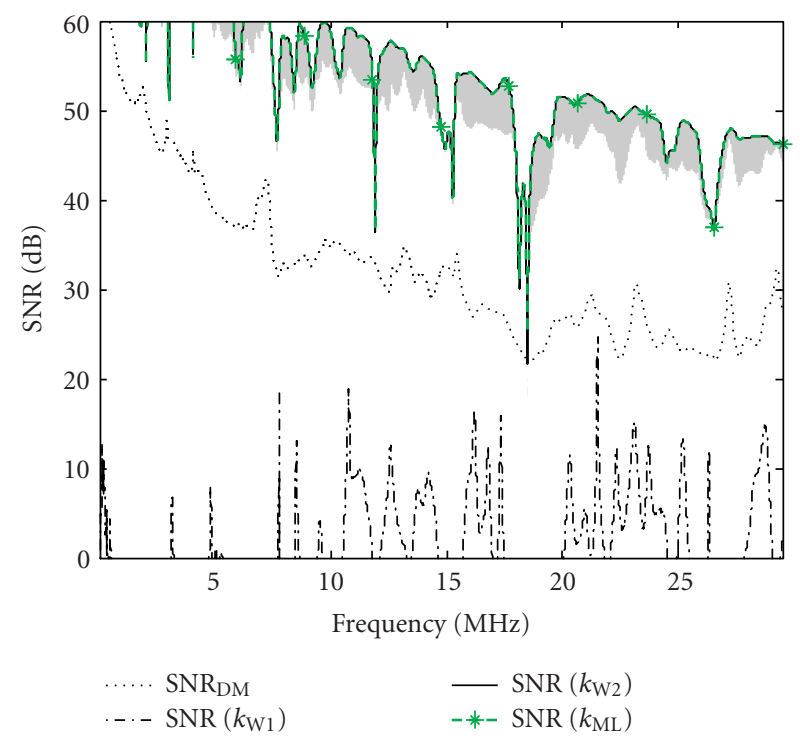

FIGURE 4: SNRs of adaptive cancellation compared to processing only the DM signal for a transmission over a loop of $300 \mathrm{~m}$ length with one FEXT source $(S=1)$ located at the same distance and transmitting with the same PSD of $-60 \mathrm{dBm} / \mathrm{Hz}$ as the far-end transmitter. The background-noise level on both DM port and CM port is $-140 \mathrm{dBm} / \mathrm{Hz}$. The grey-shaded area indicates SNR values for coefficient mismatch of up to $10 \%\left(\operatorname{SNR}\left(\mathcal{K}_{0.1}\right)\right)$.

Figure 5 shows the results for a scenario with the same parameters but with $S=2$ crosstalkers located at a distance of $300 \mathrm{~m}$ from our receiver. Both crosstalk sources transmit with the same PSD as the transmitter. On most subchannels, $\operatorname{SNR}\left(k_{\mathrm{W} 2}\right)$ exceeds $\mathrm{SNR}_{\mathrm{DM}}$. Since the canceller tries to elim- 


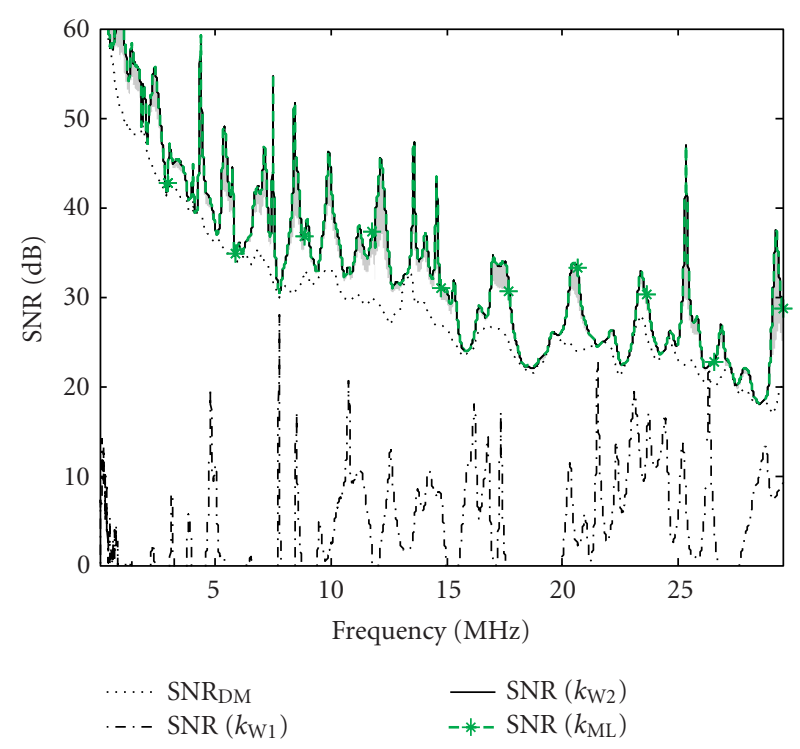

FIGURE 5: SNRs of adaptive cancellation compared to processing of DM signal only for a transmission over a loop of $300 \mathrm{~m}$ length with two FEXT sources $(S=2)$ located at the same distance and transmitting with the same PSD of $-60 \mathrm{dBm} / \mathrm{Hz}$ as the far-end transmitter. The background-noise level on both DM port and CM port is $-140 \mathrm{dBm} / \mathrm{Hz}$. The grey-shaded area indicates SNR values for coefficient mismatch of up to $50 \%\left(\operatorname{SNR}\left(\mathcal{K}_{0.5}\right)\right)$.

\subsection{Example 2: near-far scenario}

Another scenario of practical relevance is depicted in Figure 7 . We investigate the upstream transmission of customer $\mathrm{A}$, who is located at a distance of $750 \mathrm{~m}$ from the central office. The upstream transmission of customer A is mainly disturbed by strong FEXT caused by the upstream transmission of customer B, who is located at a distance of only $250 \mathrm{~m}$. This scenario represents a near-far problem often encountered in practice. Typically, there are only few customers located at a very short distance from the central office. The number of customers located at a medium distance is larger. Thus, we introduce customers $\mathrm{C}$ and D located at a distance of $750 \mathrm{~m}$ from the central office. All transmitters use a transmit PSD of $-60 \mathrm{dBm} / \mathrm{Hz}$. A trivial solution to the near-far problem is to reduce the transmit power of customer $\mathrm{B}$ - an approach that is referred to as power backoff [15]. While power backoff, applied at the transmitter of customer $\mathrm{B}$, reduces the interference for customer $\mathrm{A}$, it also limits the achievable rate of customer $\mathrm{B}$.

Figure 8 depicts the resulting SNRs for the near-far scenario. The SNR improvement due to joint DM-CM processing is marginal for subchannels below $1 \mathrm{MHz}$ since there is interference of equal strength from several sources, which the canceller cannot eliminate. However, the gain in SNR for subchannels above $1 \mathrm{MHz}$ is large since the interference caused by customer B is dominant. The improvement in this frequency range is valuable since the range overlaps with both the lower $(3-5 \mathrm{MHz})$ and the upper $(7-12 \mathrm{MHz})$ upstream band of the bandplan referred to as "997-plan," which

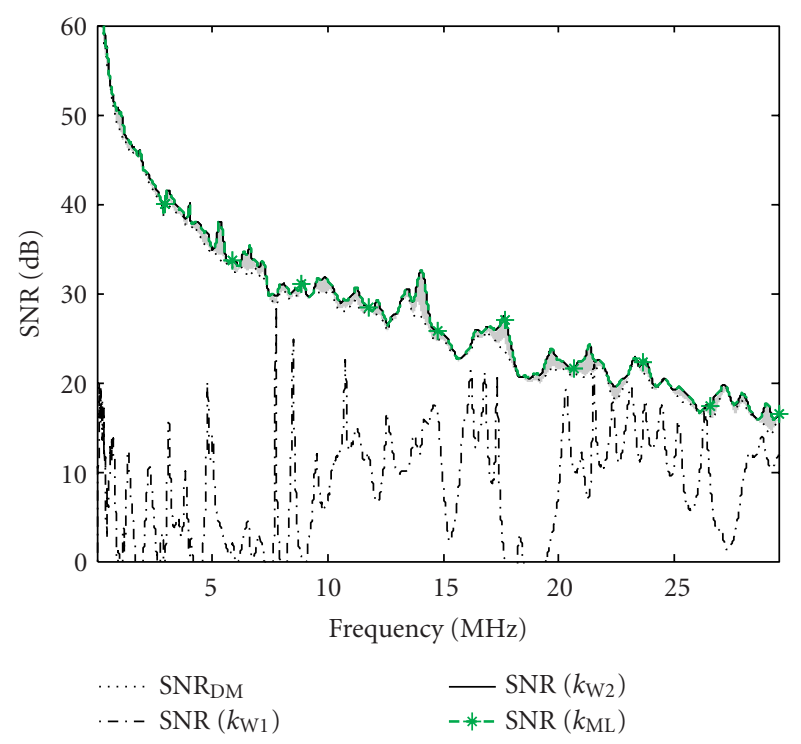

FIGURE 6: SNRs of adaptive cancellation compared to processing of DM signal only for a transmission over a loop of $300 \mathrm{~m}$ length with five FEXT sources $(S=5)$ located at the same distance and transmitting with the same PSD of $-60 \mathrm{dBm} / \mathrm{Hz}$ as the far-end transmitter. The background-noise level on both DM port and CM port is $-140 \mathrm{dBm} / \mathrm{Hz}$. The grey-shaded area indicates SNR values for coefficient mismatch of up to $100 \%\left(\operatorname{SNR}\left(\mathcal{K}_{1}\right)\right)$.

is widely used for VDSL systems [8]. For subchannels above $7 \mathrm{MHz}$, adaptive interference cancellation enables SNR values that make transmission practically feasible, which is not the case with DM-only processing. Adaptation of the coefficients in the presence of the far-end signal yields good results for subchannels above $9 \mathrm{MHz}$ since the interference caused by customer $B$ is significantly stronger than the far-end signal at these frequencies. Assumption 1 does not hold for these subchannels. Consequently, the observed behaviour is not contradictory to Proposition 2.

\section{CONCLUSIONS}

Adaptive cancellation is a viable way to exploit commonmode information in practical wireline systems since it does not require channel knowledge. A thorough performance analysis of adaptive cancellation has been presented. It was shown that adaptation of the canceller coefficients in the absence of the useful far-end signal yields an improvement in terms of throughput for a large class of practical scenarios. More importantly, adaptation in the presence of the far-end signal decreases the throughput and should thus be avoided.

The proposed subchannel interference canceller lends itself to a straightforward implementation in multicarrierbased wireline receivers. The scalar cancellers operating on subchannels can be activated individually based on the channel condition, which allows for simple adaptation and enhances robustness in case of suddenly appearing disturbers. 


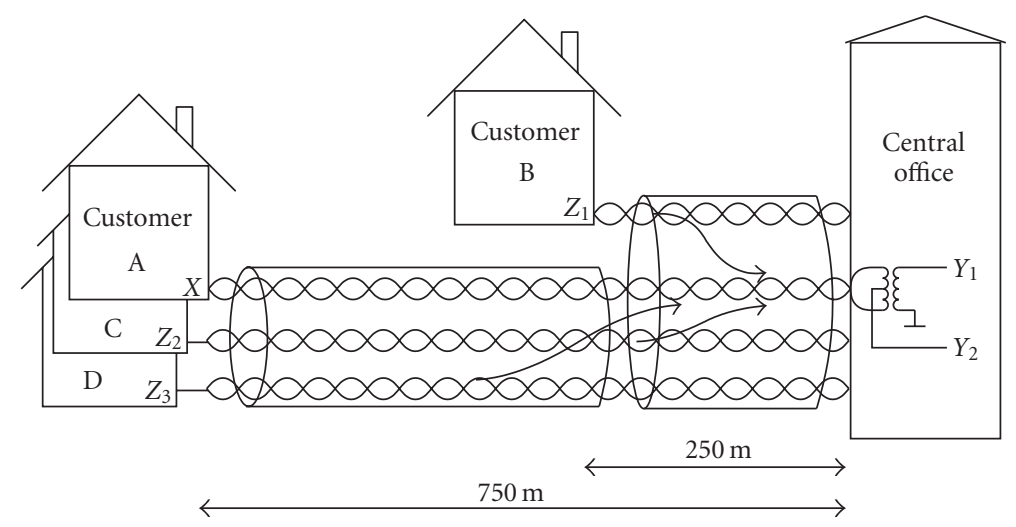

FIGURE 7: Near-far scenario: the upstream transmission of customer A is disturbed by strong FEXT from customer B, who is located closely to the central office, and by weaker FEXT from customers C and D. All FEXT sources transmit with the same PSD of $-60 \mathrm{dBm} / \mathrm{Hz}$ as the far-end transmitter of customer A. The background-noise level on both DM port and CM port is $-140 \mathrm{dBm} / \mathrm{Hz}$.

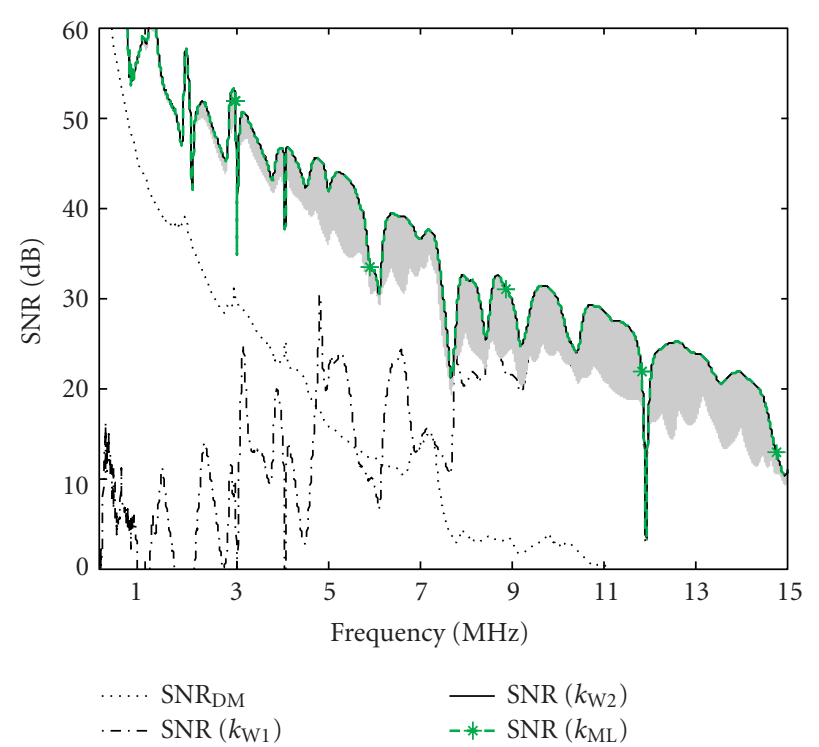

FIGURE 8: SNRs for near-far scenario. The improvement in terms of SNR for subchannels above $1 \mathrm{MHz}$ is significant. For frequencies above $7 \mathrm{MHz}$, adaptive interference cancellation yields SNR values that make transmission on these subchannel sensible, which would not be possible by processing the DM signal only. The grey-shaded area indicates SNR values for coefficient mismatch of up to $10 \%$ $\left(\operatorname{SNR}\left(\mathcal{K}_{0.1}\right)\right)$.

\section{APPENDICES}

\section{A. WIENER FILTER SOLUTION (12) FOR THE MODEL (8)}

Inserting (8) into (11) yields

$$
\begin{aligned}
k_{\mathrm{W}} & =\arg \min _{k} \mathrm{E}\left(|Y(k)|^{2}\right) \\
& =\arg \min _{k}\left(k \mathrm{E}\left(Y_{1}^{*} Y_{2}\right)+k^{*} \mathrm{E}\left(Y_{1} Y_{2}^{*}\right)+|k|^{2} \mathrm{E}\left(Y_{2} Y_{2}^{*}\right)\right) .
\end{aligned}
$$

In order to find the extremum, we set the first derivative with respect to $k$ to zero:

$$
\frac{\mathrm{d}}{\mathrm{d} k_{\mathrm{W}}}\left(k_{\mathrm{W}} \mathrm{E}\left(Y_{1}^{*} Y_{2}\right)+k_{\mathrm{W}}^{*} \mathrm{E}\left(Y_{1} Y_{2}^{*}\right)+\left|k_{\mathrm{W}}\right|^{2} \mathrm{E}\left(Y_{2} Y_{2}^{*}\right)\right) \stackrel{!}{=} 0 .
$$

Keeping in mind that $(\mathrm{d} / \mathrm{d} k) k^{*}=0$ and $(\mathrm{d} / \mathrm{d} k)|k|^{2}=k^{*}$, we obtain

$$
\mathrm{E}\left(Y_{1}^{*} Y_{2}\right)+k_{\mathrm{W}}^{*} \mathrm{E}\left(Y_{2} Y_{2}^{*}\right)=0
$$

which yields expression (12) for the Wiener filter solution in the model (8).

\section{B. PROOF OF PROPOSITIONS 1 AND 2}

Since validity of Assumption 1 is a prerequisite for Propositions 1 and 2, we begin with formalising the relations $\gg$ and $\approx$. We consider that $|v| \gg|w|$ holds if

$$
\frac{|v|}{|w|} \geq \eta
$$

for a given "large" $\eta$. A sensible choice may be $\eta=10$, which corresponds to a magnitude ratio of $20 \mathrm{~dB}$.

We consider that $|v| \approx|w|$ holds if

$$
\frac{1}{\chi} \leq \frac{|v|}{|w|} \leq \chi
$$

for a given "small" $\chi \geq 1$. A sensible choice may be $\chi=$ 2 , which corresponds to magnitude ratios in the range of $\pm 6 \mathrm{~dB}$. Hereinafter, we require that

$$
1 \leq x<\frac{\sqrt{\eta}}{2},
$$

which implies that $\eta>4$ and holds for all sensible choices of $\chi$ and $\eta$. Note that it is sufficient to prove the relations between the SNRs given by (10) and (16), since the mutual information (9) is a monotonic function of the SNR. In the proofs 
presented in the sequel, it is assumed that $S=1$. The extension for $S>1$, which is straightforward but cumbersome, does not yield any additional insight and it is thus omitted.

Proof of Proposition 1. We need to prove that $\operatorname{SNR}\left(k_{\mathrm{W} 1}\right) \leq$ $\mathrm{SNR}_{\mathrm{DM}}$, that is,

$$
\frac{\left|a+b k_{\mathrm{W} 1}\right|^{2}}{\left|c+d k_{\mathrm{W} 1}\right|^{2}+\left|n_{1}\right|^{2}+\left|n_{2} k_{\mathrm{W} 1}\right|^{2}} \leq \frac{|a|^{2}}{|c|^{2}+\left|n_{1}\right|^{2}} .
$$

The proof is laid out in three steps. First, we show that the signal power with interference cancellation using $k_{\mathrm{W} 1}$, given by $\left|a+b k_{\mathrm{W} 1}\right|^{2}$ (cf. (10)), is smaller than the signal power with DM-only reception, given by $|a|^{2}$ (cf. (16)), that is,

$$
\left|a+b k_{\mathrm{W} 1}\right|<|a|
$$

Second, we show that the resulting interference power of an interference canceller with $k_{\mathrm{W} 1}$, given by $\left|c+d k_{\mathrm{W} 1}\right|^{2}$, is larger than the interference power with DM-only reception, given by $|c|^{2}$, that is,

$$
\left|c+d k_{\mathrm{W} 1}\right|>|c|
$$

Third, we note that $\left|n_{1}\right|^{2}+\left|n_{2} k_{\mathrm{W} 1}\right|^{2} \geq\left|n_{1}\right|^{2}$, that is, that the resulting noise power with interference cancellation using $k_{\mathrm{W} 1}$ is larger than with DM-only reception.

Step 1. We start from the inequality

$$
\chi \leq \sqrt{\eta}
$$

which follows directly from (B.3). Using Assumption 1 and definitions (B.1) and (B.2), inequality (B.7) yields

$$
\begin{gathered}
\frac{|c|}{|b|} \frac{|d|}{|b|} \leq \chi^{2} \leq \eta \leq \frac{|a|}{|b|}, \\
\frac{\left|b c d^{*}\right|}{|b|^{3}} \leq \frac{|a||b|^{2}}{|b|^{3}}, \\
\frac{\left|b c d^{*}\right|}{\leq|a||b|^{2},} \\
\frac{\left|a\left(|d|^{2}+\left|n_{2}\right|^{2}\right)\right|+\left|b c d^{*}\right|}{|b|^{2}+|d|^{2}+\left|n_{2}\right|^{2}} \leq|a| .
\end{gathered}
$$

The left-hand side of (B.8) can be lower bounded by

$$
\begin{aligned}
\frac{\left|a\left(|d|^{2}+\left|n_{2}\right|^{2}\right)\right|+\left|b c d^{*}\right|}{|b|^{2}+|d|^{2}+\left|n_{2}\right|^{2}} & \geq \frac{\left|a\left(|d|^{2}+\left|n_{2}\right|^{2}\right)-b c d^{*}\right|}{|b|^{2}+|d|^{2}+\left|n_{2}\right|^{2}} \\
& =\left|a+b k_{\mathrm{W} 1}\right|
\end{aligned}
$$

where inequality and equality follow from the triangular inequality and (13), respectively. Combining (B.8) and (B.9) yields (B.5).

Step 2. It is straightforward to show that when (B.3) holds, the following inequality also holds:

$$
1 \geq \frac{2}{\eta \chi^{2}}\left(1+\frac{1}{\eta^{2}}\right)+\frac{1}{\chi^{4} \eta}
$$

Using Assumption 1 and definitions (B.1) and (B.2), inequality (B.10) yields

$$
\begin{gathered}
\frac{|a||d|}{|b|^{2}} \geq \eta \chi \geq \frac{2}{\chi}\left(1+\frac{1}{\eta^{2}}\right)+\frac{1}{\chi^{3}} \\
\geq 2 \frac{|c|}{|b|}\left(1+\frac{\left|n_{2}\right|^{2}}{|b|^{2}}\right)+\frac{|c|}{|b|} \frac{|d|^{2}}{|b|^{2}} \\
\frac{|a||d|}{|b|^{2}} \geq \frac{2|c|\left(|b|^{2}+\left|n_{2}\right|^{2}\right)}{|b|^{3}}+\frac{|c||d|^{2}}{|b|^{3}}, \\
\frac{|a||b||d|-|c|\left(|b|^{2}+\left|n_{2}\right|^{2}\right) \geq|c|\left(|b|^{2}+|d|^{2}+\left|n_{2}\right|^{2}\right),}{\left.|b|^{2}+|d|^{2}+\left|n_{2}\right|^{2}+\left|n_{2}\right|^{2}\right)} \geq|c|^{2} .
\end{gathered}
$$

The left-hand side of (B.11) can be upper-bounded by

$$
\begin{aligned}
\frac{|a||b||d|-|c|\left(|b|^{2}+\left|n_{2}\right|^{2}\right)}{|b|^{2}+|d|^{2}+\left|n_{2}\right|^{2}} & \leq \frac{\left|c\left(|b|^{2}+\left|n_{2}\right|^{2}\right)-a b^{*} d\right|}{|b|^{2}+|d|^{2}+\left|n_{2}\right|^{2}} \\
& =\left|c+d k_{\mathrm{W} 1}\right|
\end{aligned}
$$

where inequality and equality follow from the triangular inequality and (13), respectively. Combining (B.11) and (B.12) yields (B.6), which concludes the proof.

Proof of Proposition 2. We need to prove that $\operatorname{SNR}\left(k_{\mathrm{W} 2}\right) \geq$ $\mathrm{SNR}_{\mathrm{DM}}$, that is,

$$
\frac{\left|a+b k_{\mathrm{W} 2}\right|^{2}}{\left|c+d k_{\mathrm{W} 2}\right|^{2}+\left|n_{1}\right|^{2}+\left|n_{2} k_{\mathrm{W} 2}\right|^{2}} \geq \frac{|a|^{2}}{|c|^{2}+\left|n_{1}\right|^{2}}
$$

An upper bound for $\left|k_{\mathrm{W} 2}\right|$, which follows directly from (B.2), is given by

$$
\left|k_{\mathrm{W} 2}\right|=\frac{|c||d|}{|d|^{2}+\left|n_{2}\right|^{2}}<\frac{|c||d|}{|d|^{2}} \leq \chi .
$$

It is straightforward to show that when (B.3) holds, the following inequality also holds:

$$
\eta^{2}\left(1-2 \frac{\chi}{\eta}-\frac{1}{\left(1+\eta^{2}\right)^{2}}\right)-2 \frac{\chi}{\eta}-\chi^{4} \leq 0
$$

Using Assumption 1 and (B.1), we obtain from (B.15)

$$
\begin{gathered}
\frac{|c|^{2}}{\left|n_{1}\right|^{2}}\left(1-2 \frac{\chi}{\eta}-\frac{1}{\left(1+\eta^{2}\right)^{2}}\right)-2 \frac{\chi}{\eta}-\chi^{4} \leq 0 \\
|c|^{2}\left(1-2 \frac{\chi}{\eta}\right)+\left|n_{1}\right|^{2}\left(1-2 \frac{\chi}{\eta}\right) \leq \frac{|c|^{2}}{\left(1+\eta^{2}\right)^{2}}+\left|n_{1}\right|^{2}\left(1+\chi^{4}\right), \\
\frac{|a|^{2}(1-2(\chi / \eta))}{|c|^{2} /\left(1+\eta^{2}\right)^{2}+\left|n_{1}\right|^{2}\left(1+\chi^{4}\right)} \leq \frac{|a|^{2}}{|c|^{2}+\left|n_{1}\right|^{2}} .
\end{gathered}
$$


The left-hand side of (B.16) can be upper-bounded by

$$
\begin{aligned}
& \frac{|a|^{2}(1-2(\chi / \eta))}{|c|^{2} /\left(1+\eta^{2}\right)^{2}+\left|n_{1}\right|^{2}\left(1+\chi^{4}\right)} \\
& \leq \frac{|a|^{2}\left(1-2\left(|b|\left|k_{\mathrm{W} 2}\right| /|a|\right)\right)}{|c|^{2}\left(1+|d|^{2} /\left|n_{2}\right|^{2}\right)^{-2}+\left|n_{1}\right|^{2}\left(1+\left|n_{2}\right|^{2}\left|k_{\mathrm{W} 2}\right|^{2} /\left|n_{1}\right|^{2}\right)} \\
& \leq \frac{\left|a+b k_{\mathrm{W} 2}\right|^{2}}{|c|^{2}\left(1+|d|^{2} /\left|n_{2}\right|^{2}\right)^{-2}+\left|n_{1}\right|^{2}\left(1+\left|n_{2}\right|^{2}\left|k_{\mathrm{W} 2}\right|^{2} /\left|n_{1}\right|^{2}\right)} \\
& \quad=\frac{\left|a+b k_{\mathrm{W} 2}\right|^{2}}{\left|c+d k_{\mathrm{W} 2}\right|^{2}+\left|n_{1}\right|^{2}+\left|n_{2} k_{\mathrm{W} 2}\right|^{2}} .
\end{aligned}
$$

The first inequality follows from the bound (B.14), Assumption 1, and definitions (B.1) and (B.2). The second inequality follows from the triangular inequality and the equality follows from (14). Combining (B.16) and (B.17) yields (B.13), which concludes the proof.

\section{ACKNOWLEDGMENTS}

This work was supported by the European Commission and by the Swedish Agency for Innovation Systems, VINNOVA, through the IST-MUSE and the Eureka-Celtic BANITS projects, respectively.

\section{REFERENCES}

[1] A. G. Bell, "Improvement in telegraphy," Letters Patent no. 174,465, dated March, application filed February, 1876.

[2] W. Y. Chen, DSL: Simulation Techniques and Standards Development for Digital Subscriber Line Systems, Macmillan Technical, Indianapolis, Ind, USA, 1998.

[3] T. Magesacher, P. Ödling, P. O. Börjesson, and S. Shamai, "Information rate bounds in common-mode aided wireline communications," European Transactions on Telecommunications, vol. 17, no. 5, pp. 533-545, 2006.

[4] T. Magesacher, P. Ödling, P. O. Börjesson, et al., "On the capacity of the copper cable channel using the common mode," in Proceedings of IEEE Global Telecommunications Conference (GLOBECOM '02), vol. 2, pp. 1269-1273, Taipei, Taiwan, November 2002.

[5] T. Magesacher, P. Ödling, and P. O. Börjesson, "Adaptive interference cancellation using common-mode information in DSL," in Proceedings of the 13th European Signal Processing Conference (EUSIPCO '05), Antalya, Turkey, September 2005.

[6] T. H. Yeap, D. K. Fenton, and P. D. Lefebvre, "A novel common-mode noise cancellation technique for VDSL applications," IEEE Transactions on Instrumentation and Measurement, vol. 52, no. 4, pp. 1325-1334, 2003.

[7] A. H. Kamkar-Parsi, M. Bouchard, G. Bessens, and T. H. Yeap, "A wideband crosstalk canceller for xDSL using commonmode information," IEEE Transactions on Communications, vol. 53, no. 2, pp. 238-242, 2005.

[8] ETSI TM6, "Transmission and multiplexing (TM); access transmission systems on metallic access cables; very high speed digital subscriber line (VDSL)—Part 1: functional requirements," TS 101 270-1, Version 1.1.6, August 1999.

[9] T. Magesacher, W. Henkel, G. Tauböck, and T. Nordström, "Cable measurements supporting xDSL technologies," Journal e\&i Elektrotechnik und Informationstechnik, vol. 199, no. 2, pp. 37-43, 2002.

[10] ANSI T1E1.4, "Very-high-bit-rate digital subscriber line (VDSL) metallic interface-part 1: functional requirement and common specification," T1E1.4/2000-009R3, February 2001.

[11] S. M. Kay, Fundamentals of Statistical Signal Processing: Estimation Theory, Prentice-Hall, Upper Saddle River, NJ, USA, 1993.

[12] T. M. Cover and J. A. Thomas, Elements of Information Theory, John Wiley \& Sons, New York, NY, USA, 1991.

[13] K. B. Song, S. T. Chung, G. Ginis, and J. M. Cioffi, "Dynamic spectrum management for next-generation DSL systems," IEEE Communications Magazine, vol. 40, no. 10, pp. 101-109, 2002.

[14] S. Haykin, Adaptive Filter Theory, Prentice-Hall, Upper Saddle River, NJ, USA, 3rd edition, 1996.

[15] S. Schelstraete, "Defining upstream power backoff for VDSL," IEEE Journal on Selected Areas in Communications, vol. 20, no. 5, pp. 1064-1074, 2002.

Thomas Magesacher received the Dipl.-Ing. and Ph.D. degrees in electrical engineering from Graz University of Technology, Austria, in 1998 and Lund University, Sweden, in 2006, respectively. From 1997-2003, he was with Infineon Technologies (former Siemens Semiconductor) and with the Telecommunications Research Center Vienna (FTW), Austria, working on circuit design and concept engineering for communication systems. Since February 2003, he has been with Lund University, Sweden. His responsibilities include the management of national and European research projects and research cooperations with industry as well as undergraduate education. In 2006, he received a grant from the Swedish Research Council for a postdoctoral fellowship at the Department of Electrical Engineering, Stanford University, USA. His research interests include adaptive and mixed-signal processing, communications, and applied information theory.

Per Ödling was born in 1966 in Örnsköldsvik, Sweden. He received an M.S.E.E. degree in 1989, a Licentiate of Engineering degree 1993, and a Ph.D. degree in signal processing 1995, all from Luleå University of Technology, Sweden. In 2000, he was awarded the Docent degree from Lund Institute of Technology, and in 2003 he was appointed Professor there. From 1995, he was an Assistant Professor at Luleå University of Technology, serving as Vice Head of the Division of Signal Processing. In parallel, he consulted for Telia $\mathrm{AB}$ and ST-Microelectronics, developing an OFDM-based proposal for the standardisation of UMTS/IMT-2000 and VDSL for standardisation in ITU, ETSI, and ANSI. Accepting a position as Key Researcher at the Telecommunications Research Center Vienna in 1999, he left the arctic north for historic Vienna. There, he spent three years advising graduate students and industry. He also consulted for the Austrian Telecommunications Regulatory Authority on the unbundling of the local loop. He is, since 2003, a Professor at Lund Institute of Technology, stationed at Ericsson AB, Stockholm. He also serves as an Associate Editor for the IEEE Transactions on Vehicular Technology. He has published more than forty journal and conference papers, thirty-five standardisation contributions, and a dozen patents.

Per Ola Börjesson was born in Karlshamn, Sweden in 1945. He received his M.S. degree in electrical engineering in 1970 and his Ph.D. degree in telecommunication theory in 1980, both from Lund Institute of Technology (LTH), Lund, Sweden. In 1983, he 
the degree of Docent in Telecommunication Theory. Between 1988 and 1998, he was Professor of Signal Processing at Luleå University of Technology. Since 1998, he is a Professor of Signal Processing at Lund University. His primary research interest lies in highperformance communication systems, in particular, high-data-rate wireless and twisted pair systems. He is presently researching signal processing techniques in communication systems that use orthogonal frequency-division multiplexing (OFDM) or discrete multitone modulation (DMT). He emphasises the interaction between models and real systems, from the creation of application-oriented models based on system knowledge to the implementation and evaluation of algorithms. 